\title{
On the Failure and Fracture of Polymer Foam Containing Discontinuities
}

\author{
Addis Kidane \\ Department of Mechanical Engineering, University of South Carolina, 300 Main Street, Columbia, SC 29208, USA \\ Correspondence should be addressed to Addis Kidane; kidanea@mailbox.sc.edu
}

Received 19 June 2013; Accepted 4 August 2013

Academic Editors: Y. Hiraoka and K. Hokamoto

Copyright (C) 2013 Addis Kidane. This is an open access article distributed under the Creative Commons Attribution License, which permits unrestricted use, distribution, and reproduction in any medium, provided the original work is properly cited.

An experimental study is performed to investigate the tensile failure and fracture behavior of polymer foam containing discontinuities. PVC corecell foam, series A800 and A1200 is used for the investigation. Unnotched dog-bone specimen and specimens with center hole and edge cracks are tested under uniaxial tensile loading. Series of experiments are conducted at different defect size to width ratios, and the effect of the defect size on the net-section tensile strength of the foam is investigated. A fracture study is also conducted, and the effect of density and loading rate on the fracture behavior of foam is investigated. A minimal notch-strengthening effect is observed in specimens with center hole, and a notch-weakening effect is observed in specimen with edge notches. Furthermore, the fracture toughness increases with the increase in the foam density and decreases with the increase in loading rate.

\section{Introduction}

Polymer foams are widely used in lightweight structures, such as core material for sandwich structures, due to their superior blast mitigation and impact resisting behavior. Currently, sandwich structures are among the lightweight structures widely used in aerospace, navy, and other related industries. Polymer foams have shown promising results as a core material for sandwich structures due to their high energy absorption capabilities, especially in the case of impact loading $[1,2]$. There are well documented studies on the compressive properties and energy absorbing behavior of these materials [3]. It is shown that the structural response of a polymer foam strongly depends on the foam density, cell microstructure, and solid polymer properties [3]. Recent developments in the manufacturing processes of polymer foams contribute to the expansion of these materials in structural applications. Some of the applications involve cutout and holes. It is well understood that, in the case of a structure with a fully dense material, the presence of cracks, notches, and holes results in a stress concentration that leads to fracture.
To understand these phenomena, the fracture behavior of polymers has been studied by different researcher including the early work of McIntyre and Anderton [4] and others [5-7]. McIntyre and Anderton [4] investigated the fracture toughness of a rigid polyurethane foam over a range of densities and found that the fracture toughness increases with density. The fatigue crack growth in the polyurethane foam has been investigated by Noble and Lilley [8]. The fracture process occurring in rigid, closed-cell polyurethane foam has been investigated by Cotgreave and Shortall [9], and the morphology and fracture behavior of polyurethane foams reinforced by short chopped fibers have been investigated by Cotgreave and Shortall [10]. The effect of different parameters such as matrix composition [11], microstructure and morphology [12], density [13, 14], strain rate [14], and inclusions [15] on the mechanical behavior of foams have been also investigated. Recently, Marsavina et al. [16] present a comparison between static and dynamic fracture toughness of a polymer and found that the dynamic fracture toughness is higher than the quasi-static fracture toughness. On the contrary, very recently Rizov [17] showed that the dynamic fracture toughness of rigid foam is between $22.4 \%$ and $32 \%$, 


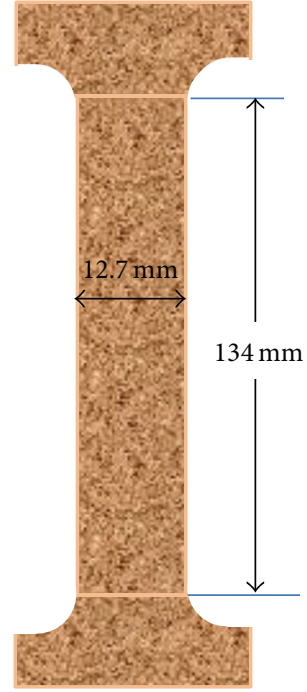

(a)

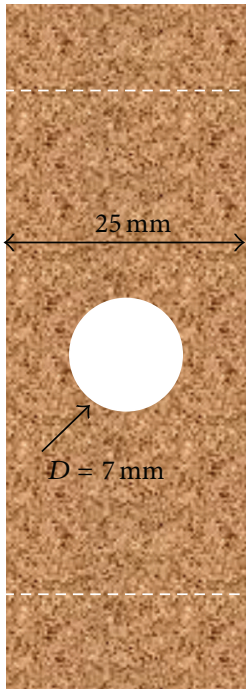

(b)

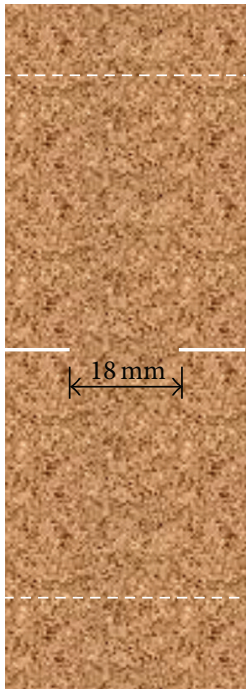

(c)

FIGURE 1: Schematic drawing and geometry of the tensile specimens for A800 and A1200 foam, (a) dog-bone specimen without a defect (DB), (b) with a center hole $(\mathrm{CH})$, and (c) with an edge crack (EC).

lower than the static value. As discussed above, though the density has a direct correlation with the facture toughness, a detailed investigation is required to clearly understand the effect of strain rate on the fracture properties of polymer foams.

There are also reports in the literature dealing with the effect of defects such as central cracks, edge notches, and holes on the tensile strength of both the polymer and the metal foams [18-22]. Most of the studies prevailed that for the defects smaller than the cell size of the foam, the tensile strength is not affected by the defects. On the other hand, there is inconsistent report on the effect of defects on the net-section tensile strength of foams, for defects larger than the foam cell size. Some of the studies indicated that the netsection strength of foams with defects larger than the foam cell size is higher than the virgin specimen, resulting in a notch-strengthening effect $[18,19,22]$. Some other studies reported that the net-section strength of a specimen with defects is lower than the virgin specimen, indicating a notchweakening effect [20-22], and the rest of the studies indicated that the net-section strength is insensitive to the defects [21].

In this paper, the failure and fracture property of PVC foam with discontinuities is investigated. The effect of defects size and geometry on the tensile failure properties of PVC foam is discussed. Furthermore, the fracture properties as a function of density and loading rate are also presented.

\section{Material and Specimen Geometry}

The foam material used in the present study is Corecell A series styrene foam, manufactured by Gurit SP Technologies. These materials are widely used for marine sandwich composite due to their large shear elongation behavior. Selective material properties for A800 and A1200 Corecell foam used
TABLE 1: Selected mechanical properties of A800 and A1200 Corecell foam (http://www.gurit.com).

\begin{tabular}{lccc}
\hline Material & $\begin{array}{c}\text { Nominal density } \\
\left(\mathrm{kg} / \mathrm{m}^{3}\right)\end{array}$ & $\begin{array}{c}\text { Shear modulus } \\
(\mathrm{MPa})\end{array}$ & $\begin{array}{c}\text { Shear } \\
\text { elongation }(\%)\end{array}$ \\
\hline A800 & 150 & 47 & 50 \\
A1200 & 176 & 60 & 47 \\
\hline
\end{tabular}

in the present study obtained from the manufacturer's data source (http://www.gurit.com) are listed in Table 1.

For the tensile experiment, three different specimen geometries, dog-bone specimen without a defect (DB), with an edge crack (EC), and with a center hole $(\mathrm{CH})$, as shown in Figure 1, are machined from a $25 \mathrm{~mm}$ thick foam plate. The gage lengths are $134 \mathrm{~mm}$, the effective widths are $18 \mathrm{~mm}$, and the thicknesses are $6 \mathrm{~mm}$.

For the fracture experiment, single edge notched bend (SENB) specimens, as shown in Figure 2, are prepared from A800 and A1200 foam sheets according to ASTM E3999. Two different size samples are used in the fracture experiment. The span length for sample Type 1 is $184 \mathrm{~mm}$, the width is $W=$ $50.8 \mathrm{~mm}$, the thickness is $B=25.4 \mathrm{~mm}$, and the initial crack length is $a=20.32 \mathrm{~mm}$. The crack is first machined with $1 \mathrm{~mm}$ thick blade, and later the artificial crack is extended with a razor blade. For the dynamic fracture experiment, a smaller specimen (sample type 2) with a dimension of $1 / 3.6$ th of the quasi-static specimen is used.

\section{Experimental Procedure}

3.1. Tensile Experiment. For the tensile experiment, a table top Instron machine is used in a displacement control mode at a speed of $1 \mathrm{~mm} / \mathrm{min}$, and the load is recorded directly by the load cell. To measure the strain fields around the gage 


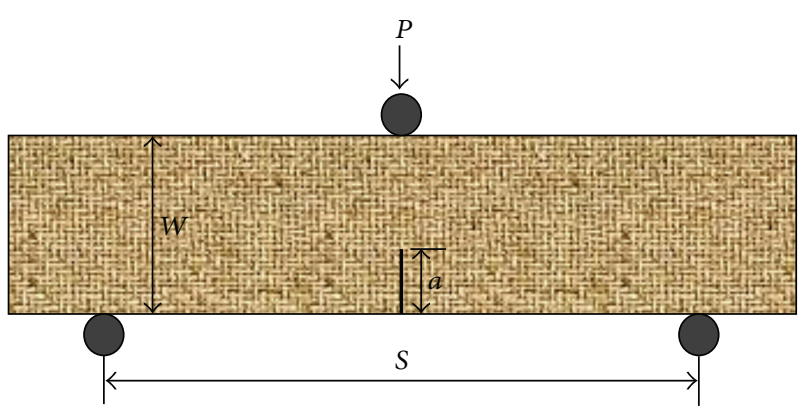

FIGURE 2: Schematic drawing and geometry of a single notched three-point bending specimen.

length, a $2 \mathrm{D}$ digital image correlation (DIC) technique is used. First, the specimens are painted with a very thin layer of white paint, and then a black paint is arbitrarily speckled on the top of the white paint, which gives a random black and white contrast pattern. Digital images of the sample, during deformation, at several deformation steps are taken using a high speed CCD camera at a resolution of $2048 \times 2048$ pixels. First, the displacement and the strain field around the gage length are calculated from the acquired image using Vic2D commercial software, and later, the strains around the required field are extracted.

The tensile load measured directly through the load cell is used to calculate the tensile stress, and the average of strains along the gage section obtained from the DIC is used to calculate the corresponding average strain. The net-section strength, in the case of the specimens with notches and a circular hole, is defined as the maximum tensile load divided by the ligament area in the section with the defect. It is worth to mention that for each type of specimen a minimum of three experiments are conduced to check repeatability.

3.2. Three-Point Bending. The stress intensity factor $K_{I}$ is obtained experimentally using a single edge notched specimen in three point-bending experiments. An Instron machine is used in a displacement control mode at a cross head speed of $1 \mathrm{~mm} / \mathrm{min}$, and the loading data is recorded directly by the load cell. Digital images of the sample at several deformation steps are taken using a high speed CCD camera. A 2D DIC technique is used to obtain the displacement and strain fields. Figure 3 shows a typical speckle pattern of a three-point bend specimen near the crack tip.

The stress intensity factor $K_{I}$ is calculated using three different techniques: (1) from a critical load in the loaddisplacement curve using the linear elastic fracture mechanics theory, (2) the stress intensity factor formulation using a single strain data point with a three-parameter solution described by Dally and Sanford [23], and (3) an overdeterministic approach proposed by Berger and Dally [24]. The detailed analysis of the above techniques can be obtained in the literature [23,24], and only a brief description is presented below.

3.2.1. Load Displacement. The stress intensity factor $K_{I}$ can be calculated from the critical load in the load-displacement

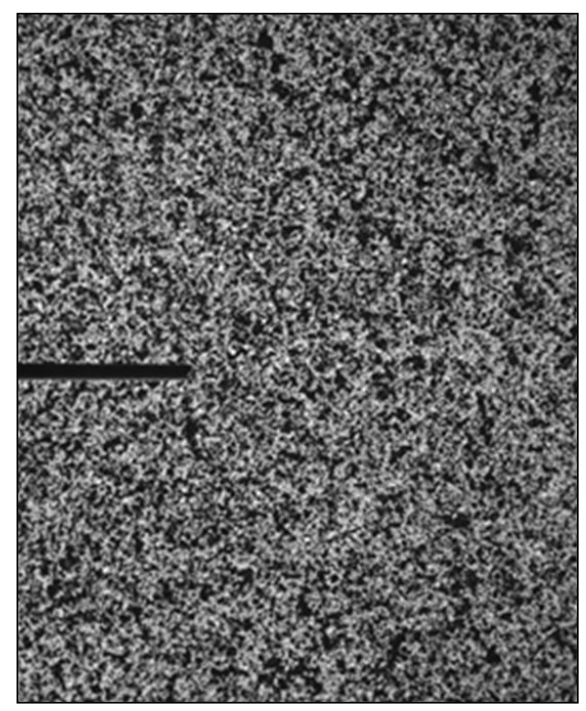

FIGURE 3: Typical speckle pattern near the crack tip region of the SENB specimen.

curve and shape function using the linear elastic fracture mechanics relation given by (1)-(2) [25].

$$
\begin{aligned}
K_{I} & =\sigma \sqrt{\pi a} f\left(\frac{a}{W}\right), \\
\sigma & =\frac{3}{2} \frac{p S}{B W^{2}},
\end{aligned}
$$

where $p$ is the critical load, a is the crack length, $B$ is the specimen thickness, $W$ is the specimen width, $S$ is the span, and $f(a / W)$ is the geometric shape factor and can be given as

$$
\begin{aligned}
F\left(\frac{a}{W}\right)= & 1.090-1.735\left(\frac{a}{W}\right)+8.20\left(\frac{a}{W}\right)^{2} \\
& -14.18\left(\frac{a}{W}\right)^{3}+14.57\left(\frac{a}{W}\right)^{4}
\end{aligned}
$$

3.2.2. Single Point Three-Parameter Solution. The second approach is a method first proposed by Dally and Sanford [23] where they use a strain gage to determine the mode I stress intensity factor. In this method, a single or two-strain gages will be placed on a region where the strain fields can be described by a three- or four-parameter solution. For a three-parameter solution, only a single strain gage is required positioned near to the crack tip and oriented at a specific direction. The orientation angle $\alpha$ of the strain gage is a function of the materials Poisson's ratio $(v)$ and can be given as,

$$
\cos (2 \alpha)=-\frac{(1-\nu)}{(1+\nu)}
$$

The location of the strain gage from the crack tip direction can be represented by the radius, $r$, and the angle, $\theta$, as shown in 
Figure 4. This angle is unique and related to $\alpha$ as shown by (4):

$$
\tan \left(\frac{\theta}{2}\right)=\cot (2 \alpha)
$$

The stress intensity factor can be given as

$$
\begin{aligned}
K_{I}= & E \sqrt{2 \pi r} \varepsilon_{r r} \\
\times & \left(\operatorname { c o s } \frac { \theta } { 2 } \left[1+\sin \frac{\theta}{2} \sin \frac{3 \theta}{2}\right.\right. \\
& \left.\left.-v\left(1-\sin \frac{\theta}{2} \sin \frac{3 \theta}{2}\right)\right]\right)^{-1},
\end{aligned}
$$

where $E$ and $v$ are the Young's modulus and Poison's ratio of the material and $\varepsilon_{r r}$ is the strain at $r$ and $\theta$ in the radial direction.

This technique is easy and involves only a single strain gage measurement. However, it suffers from two main drawbacks. First, the accuracy is highly sensitive to the orientation of the strain gage. A small deviation of the strain gage changes the result a lot. Second, as only the first three terms are used, the strain gage has to be placed near to the crack tip to avoid the effect of the higher order terms. On the other hand the mounting the strain gages too close to the crack tip will be affected by the plastic zone, and hence a prior knowledge of the plastic zone is essential.

To minimize the error associated with the strain gage positioning, a digital image correlation technique is used. DIC enables taking a series of strain points, and hence knowledge of the plastic zone prior to the experiment is not required. First, the full strain field around the crack tip is measured, and then strain data at different radii are considered and used to calculate the stress intensity factor. Digital images of the sample at several deformation steps are taken using a CCD camera. In these fracture tests, the foam specimen undergoes only small amount of deformation before failure, and the out-of-plane deformation is negligible. Therefore, the tests are considered as a $2 \mathrm{D}$, and only a single camera is used to record the images. A commercial digital image correlation software Vic-2D is used to extract strain fields, and (5) is used to calculate fracture intensity factor.

3.2.3. An Overdeterministic Approach. To eliminate the constraints imposed in the technique described in Section 3.2.2, an overdeterministic approach was proposed by Berger and Dally [24]. The technique allows considering a larger number of strain points at a location far from the crack tip where the plastic zone correction is negligible and to account for the effect of higher order terms. Unfortunately, the techniques require a larger number of strain gages, which makes it a very difficult and costly technique as the strain gages are not reusable. Furthermore, the strain gages give a strain only in one direction that requires the strain field equations to be transformed to radial directions. In the present study, strains are obtained using DIC. Unlike the strain gages, the DIC technique is full field and makes choosing strains at any location easy. Furthermore, since the 3D strain fields

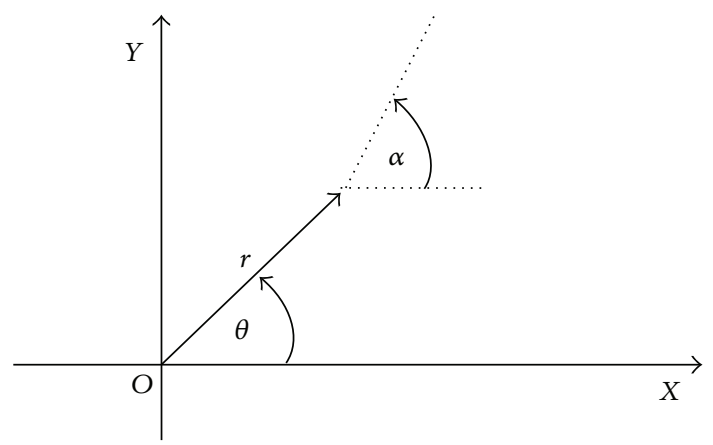

FIGURE 4: Strain gage position with respect to the crack tip position.

at any point are obtained a transformation of the strain field equations to the radial direction is not required. The strain field for an opening mode crack in a six-term series representation can be written as

$$
\begin{aligned}
E \varepsilon_{x x}= & A_{0} r^{-1 / 2} \cos \left(\frac{\theta}{2}\right) \\
& \times\left((1-\nu)-(1+\nu) \sin \left(\frac{\theta}{2}\right) \sin \left(\frac{3 \theta}{2}\right)\right) \\
& +2 B_{0}+A_{1} r^{1 / 2} \cos \left(\frac{\theta}{2}\right) \\
& \times\left((1-\nu)+(1+\nu)\left(\sin \left(\frac{\theta}{2}\right)\right)^{2}\right)+2 B_{1} r \cos (\theta) \\
& +A_{2} r^{3 / 2}\left((1-\nu) \cos \left(\frac{3 \theta}{2}\right)-\left(\frac{3}{2}\right)\right. \\
& \left.\quad \times(1+\nu) \sin (\theta) \sin \left(\frac{\theta}{2}\right)\right) \\
& +2 B_{2} r^{2}\left((1+\nu)(\sin (\theta))^{2}+\cos (2 \theta)\right),
\end{aligned}
$$

$$
\begin{aligned}
E \varepsilon_{y y}= & A_{0} r^{-1 / 2} \cos \left(\frac{\theta}{2}\right) \\
& \times\left((1-v)+(1+v) \sin \left(\frac{\theta}{2}\right) \sin \left(\frac{3 \theta}{2}\right)\right) \\
& -2 B_{0} v+A_{1} r^{1 / 2} \cos \left(\frac{\theta}{2}\right) \\
& \times\left((1-v)-(1+\nu)\left(\sin \left(\frac{\theta}{2}\right)\right)^{2}\right)-2 B_{1} v r \cos (\theta) \\
& +A_{2} r^{3 / 2}\left((1-v) \cos \left(\frac{3 \theta}{2}\right)+\left(\frac{3}{2}\right)\right. \\
& \left.\quad \times(1+v) \sin (\theta) \sin \left(\frac{\theta}{2}\right)\right) \\
& +2 B_{2} r^{2}\left((1+\nu)(\sin (\theta))^{2}-v \cos (2 \theta)\right),
\end{aligned}
$$




$$
\begin{aligned}
\mu \gamma_{x y}= & \left(\frac{1}{2}\right) A_{0} r^{-1 / 2} \sin \left(\frac{\theta}{2}\right) \cos \left(\frac{3 \theta}{2}\right) \\
& -\left(\frac{1}{2}\right) A_{1} r^{1 / 2} \sin (\theta) \cos \left(\frac{\theta}{2}\right)-B_{1} r \sin (\theta) \\
& -\left(\frac{3}{2}\right) A_{2} r^{3 / 2} \sin (\theta) \cos \left(\frac{\theta}{2}\right)-2 B_{2} r^{2} \sin (2 \theta)
\end{aligned}
$$

In the previous equations, the coefficients $A_{0}, A_{1}, A_{2}, B_{0}, B_{1}$ and $B_{2}$, are unknown and have to be solved by considering large data points around the crack tip and using a leastsquare approach [24]. Once the coefficients are determined, the mode I stress intensity factor can be determined from (9):

$$
K_{I}=A_{0} \sqrt{2 \pi r}
$$

3.3. Dynamic Fracture. The dynamic fracture behavior of the foam discussed above is investigated using the modified split Hopkinson pressure bar (SHPB). The modified Split Hopkinson pressure bar has been widely used to determine the dynamic fracture properties in metals and other high impedance materials $[26,27]$. The apparatus mainly consists of an incident bar, a striker bar, and a pressure gun. To generate a well-defined loading pulse, the same material that is used for the incident bar is used for the striker bar. Two strain gages are attached in the middle of the incident bar diametrically opposite to one another. During loading, the specimen is sandwiched between the incident bar and the rigid frame in a three-point bending arrangement, and the striker bar is propelled towards the incident bar using an air-operated gun. The impact generates a compressive stress wave in the bar which propagates toward the bar-specimen interface. When the wave reaches the specimen, some of the wave is reflected back, and part of the wave is transmitted into the specimen. The incident and reflected strain signals are recorded by the strain gages attached in the bar. These signals are used to calculate the force applied to fracture the sample. By neglecting the inertia effect, the fracture toughness can be calculated using the fracture force and (1)-(2).

To reduce the impedance mismatch between the test specimen and the pressure bar, in the present experiment, polycarbonate projectile and incident bar are used. Since polycarbonate is a viscoelastic material, the conventional SHPB analysis cannot be used [28-31]. More importantly, the strain signals recorded at the strain gage locations cannot be directly used to find the force at the specimen-bar interface. The analysis should account for the attenuation and the dispersion of stress pulse in the polymeric bars [28-31]. The theoretical relation given by Sharma and Shukla [30] is used to extract the force at the specimen-bar interface by accounting for dispersion and attenuation.

\section{Results and Discussions}

4.1. Tensile Behavior. A typical stress-strain plot for A800 and A1200 polymer foam unnotched dog-bone specimen

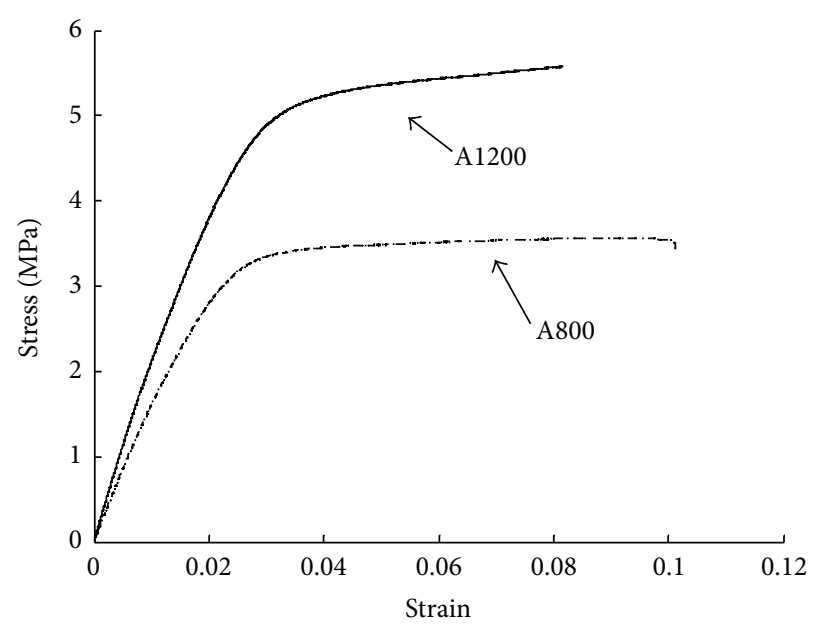

Figure 5: Typical tensile stress-strain plot for A800 and A1200 foam unnotched dog-bone specimen.

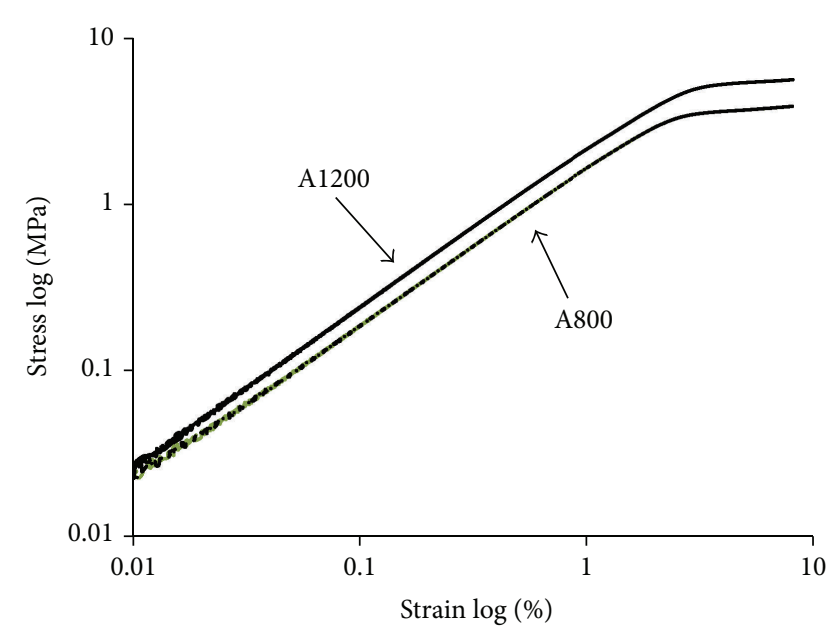

FIGURE 6: Stress-strain curve in a double logarithmic plot for A800 and A1200 foam under uniaxial tensile loading.

subjected to tensile load is plotted in Figure 5. The specimen showed a typical strain stress plot of a soft material, yielding followed by hardening and failure. The strain work-hardening coefficient is calculated from the uniaxial stress strain data obtained from the dog-bone sample. Figure 6 shows a double logarithmic plot of stress-strain for A800 and A1200 foam under tension. The slope of the curve in the plastic regime gives the work-hardening exponent, " $n$." The average workhardening exponent, $n$, for A800 and A1200 foam is 0.126, which is in the range of typical values for polymers.

On the other hand, the transverse and the longitudinal strains of the un-notched sample obtained from the $2 \mathrm{D}$ digital image correlation are used to calculate the Poisson's ratio. Figure 7 shows the plot of the transverse strain versus the longitudinal strain for A800 foam under tension. The Poisson's ratio, $v$, which is the slope of the curve shown in the Figure 7, is about 0.215 . A series of tensile experiments are conducted, and the average yielding stress and failures 


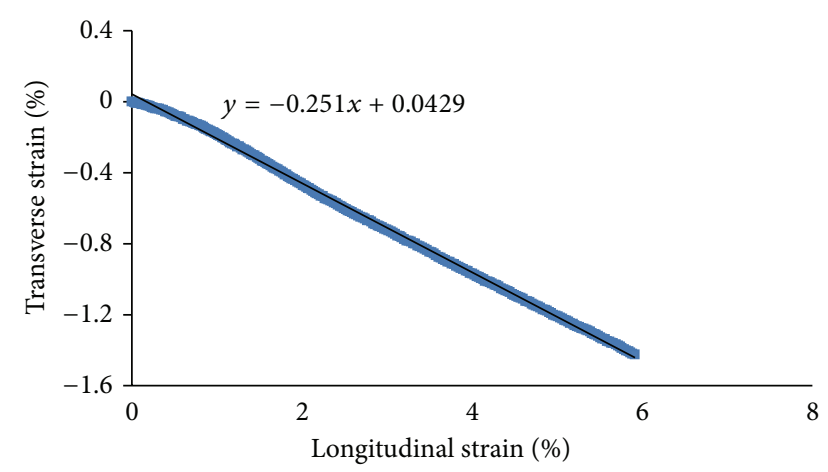

FIGURE 7: Typical transverse strain versus longitudinal strain for the A800 polymer foam under tension.

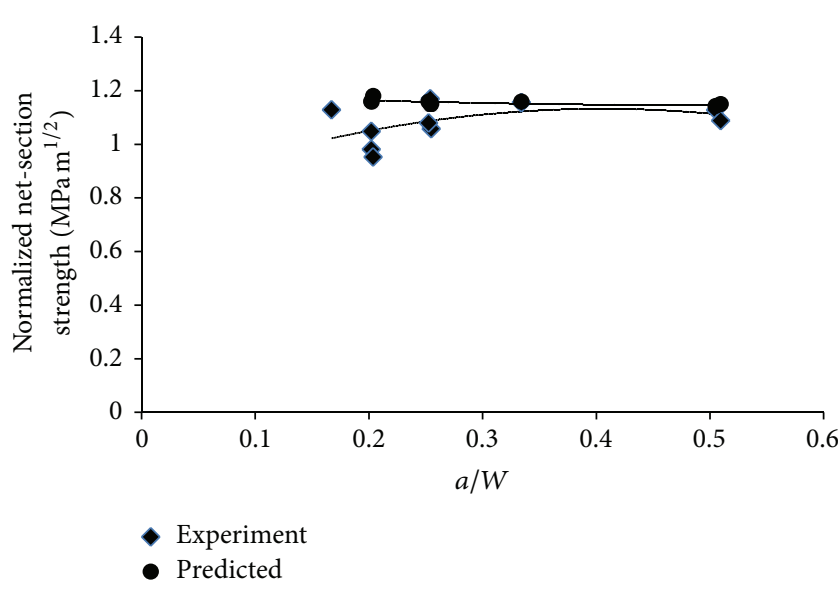

FIGURE 8: Normalized net-section strength as a function of normalized hole diameter for A800 foam specimen with center-hole.

stress for A800 foam are about 3.49 MPa and 3.89 MPa and for A1200 foam are about 5.12 MPa and 5.61 MPa, respectively.

4.2. Effect of Defects Size on the Net-Section Strength. Unlike the un-notched specimen, in the case of specimens with defects, the failure is abrupt and occurrs without showing any hardening effect. The average net-section tensile strength for A800 foam with center hole and center crack is about 3.91 MPa and with edge notches is about $3.82 \mathrm{MPa}$, which are very close the virgin specimen. To further understand the effect of defects size on the tensile strength of foams, tensile tests at different hole-diameter to specimen width ratio are performed. Figure 8 shows the net-section strength as a function of hole-diameter to specimen-width ratio for A800 foam sample with a circular hole at the center. As shown in the figure, the net-section strength slightly increases as the diameter to width ratio increases. This variation and strengthening effect can be related to a relocation of the weak plane by the defects.

According to Andrews and Gibson [18], this net-section strength is a function of the notch depth, the specimen width,

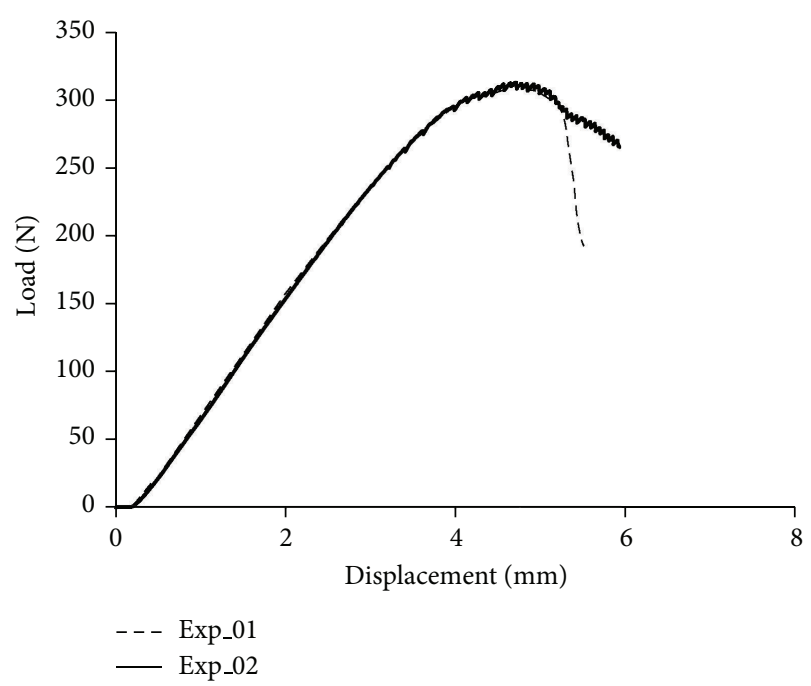

Figure 9: Typical load displacement plot for the A1200 foam threepoint bending test.

and the cell size and the normalized net-section strength can be calculated as

$$
\frac{\sigma_{\text {net }}}{\sigma_{\max }}=1+\left(\frac{d}{W}\right)\left(\frac{1}{1-a / W}\right)
$$

where $d$ is the foam cell size, $W$ is the width of the specimen, and $a$ is notch depth.

As shown in (10), for a known value of the foam cell size, the normalized net-section strength can be calculated as a function of the hole-diameter size to width ratio. For A800 foam, the cell size is about $3 \mathrm{~mm}$, and the normalized net section strength is calculated accordingly. The theoretical model agrees with the current experiment in a certain region of $a / W$ ratio and starts to deviate as the $a / W$ ratio decreases as shown in Figure 8.

\subsection{Fracture Behavior}

\subsubsection{Quasistatic Fracture}

From Critical Load History. A typical load-deflection curve for A1200 foam obtained from SENB experiment is plotted in Figure 9. The stress intensity factor is calculated using (1) and the critical load from the load-deflection curve. The average quasi static stress intensity factors $K_{I}$ for A1200 foam obtained from three similar experiments is $0.53 \mathrm{MPa} \mathrm{m}^{1 / 2}$, with an error in the rage of -0.011 to +0.028 .

Using a Single Strain Data Point. The displacement and the strain fields around the crack tip are calculated from the acquired image using a commercial software vic-2D by the correlated soln. Figure 10 shows the strain contour for A1200 foam around the crack tip just before the crack propagates. The Poison's ratio for A1200 foam is 0.22 and results in $\alpha=$ 64.68 and $\theta=78.65$. Using these two angles, multiple points at different radii are considered and (5) is used to calculate the stress intensity factor. The stress quasi-static intensity factor 


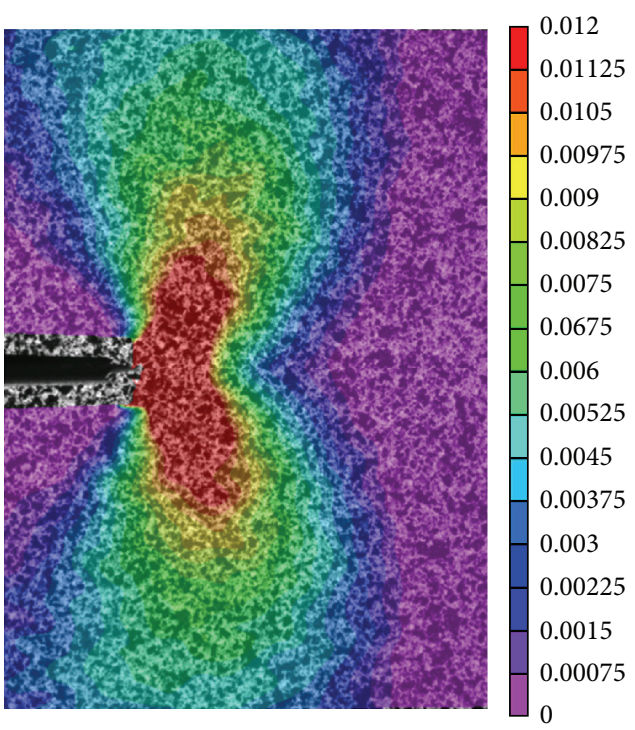

FIGURE 10: Opening mode strain field near the crack tip for A1200 foam.

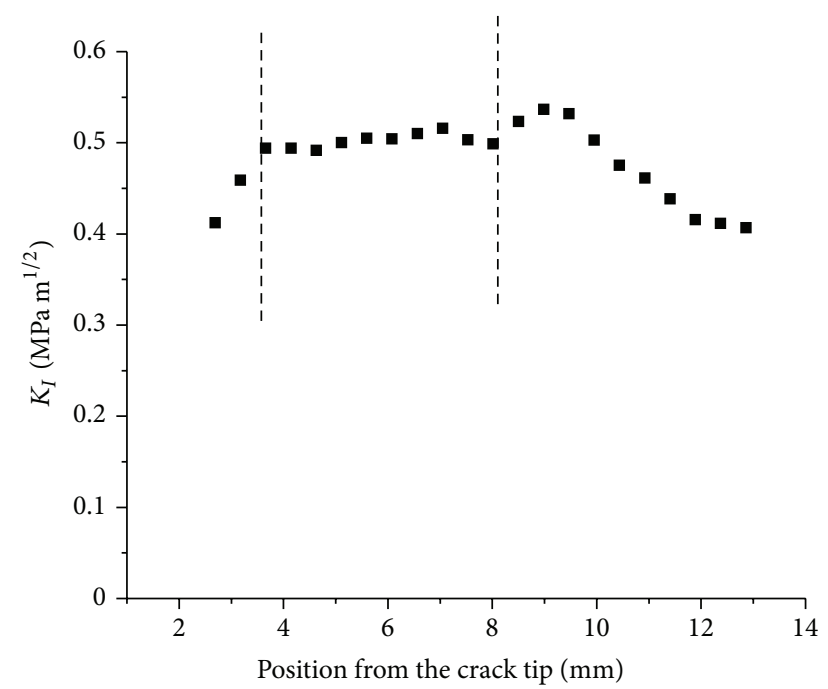

FIGURE 11: Quasistatic stress intensity factor as a function of distance from the crack tip $(r)$ for A1200 foam.

as a function of distance from the crack-tip $(r)$ for A1200 foam is presented in Figure 11. In the region where the first term is dominant but outside the non-linear crack-tip region, the result is consistent and reputable. On the other hand, in the region near to the crack tip and far away from the region where the first term is dominated, the result underestimates the value of $K_{I}$. Later it is found that, the plastic zone for this geometry and material is about $3 \mathrm{~mm}$ and the region between $r=3 \mathrm{~mm}$ to $9 \mathrm{~mm}$ gives an acceptable result for mode $I$ stress intensity factor. As it is shown in the figure, the average fracture initiation toughness is about $0.5 \mathrm{MPa} \mathrm{m}^{1 / 2}$.

Using Overdeterministic Approach. Finally, (6)-(8) are used to calculate the stress intensity factor using multiple strain

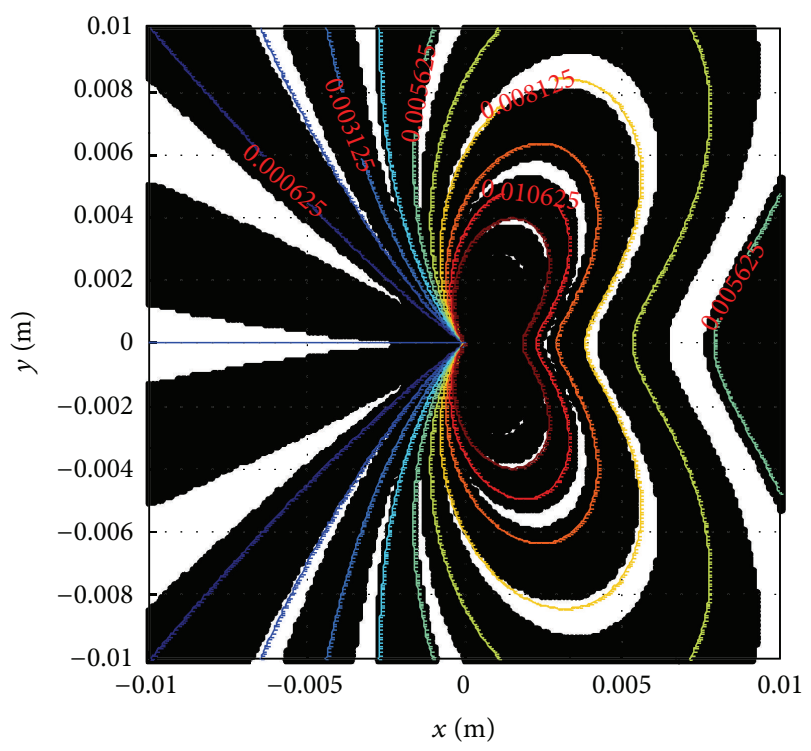

FIGURE 12: The strain field $e_{y y}$ regenerated using the calculated $K_{I}$ for A1200 foam.

points. A total of 30 data points are considered, and $K_{I}$ is calculated using (6)-(8) and the least square approach. The value of $K_{I}$ is very close to the one obtained in the previous two cases, with average value of $K_{I}=0.52 \mathrm{MPa} \mathrm{m}^{1 / 2}$. Using the calculated stress intensity factor and other constants, the strain field is regenerated as shown in Figure 12. The strain field, $e_{y y}$, regenerated from the calculated $K_{I}$ and the corresponding coefficients matches well with the original strain field obtained from the DIC shown by Figure 11.

4.3.2. Effect of Density and Loading Rate. As shown above, the fracture intensity factor results from three different methods match very well. Here, the effect of density and loading rate on the fracture behavior of foam is investigated. As stated earlier in Section 2, smaller specimens are used in the case of dynamic experiment. The two samples are proportional, and the geometry ratio between the quasi-static and dynamic samples is 3.6. Typical incident and reflected force signals obtained from the modified SHPB test shown in Figure 13. These signals are used to calculate the force at the barspecimen interface, which is the fracture force shown by Figure 14. Assuming the inertial effect is negligible, (1)-(2) are used to calculate the fracture intensity factor. Different samples with different initial crack length are investigated, and the results are presented in Figure 15. It is found that the dynamic fracture toughness for both A800 and A1200 is lower than the quasi-static value and could be due to the brittle nature of the polymer foam under dynamic loading. Furthermore, the fracture toughness increases as the density increases, which agrees well with the literature [4].

\section{Conclusion}

An experimental investigation on the effects of holes, cracks, and notches on the tensile properties of a polymer foam 


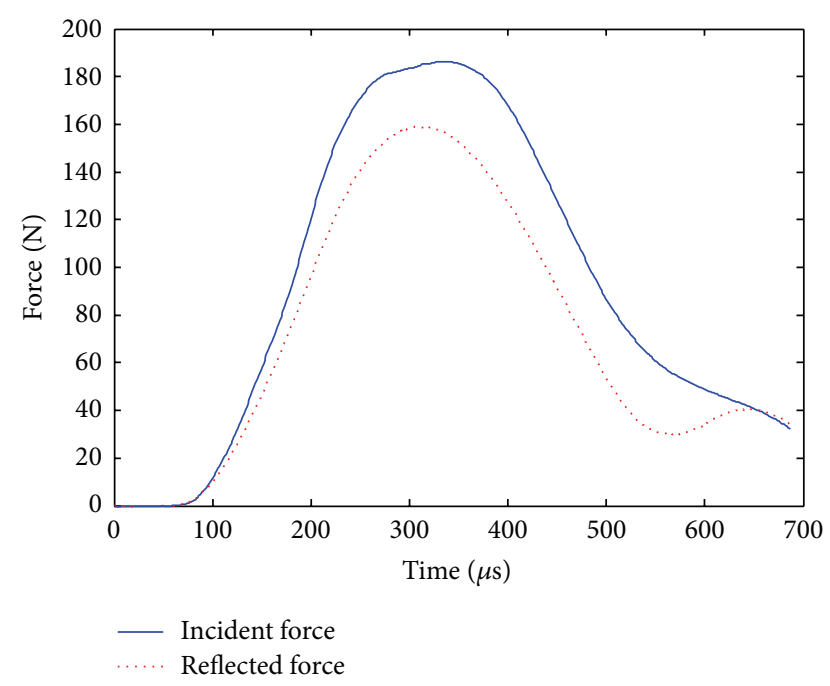

Figure 13: Typical incident and reflected forces for A1200 foam under a dynamic three-point bend test using modified SHPB.

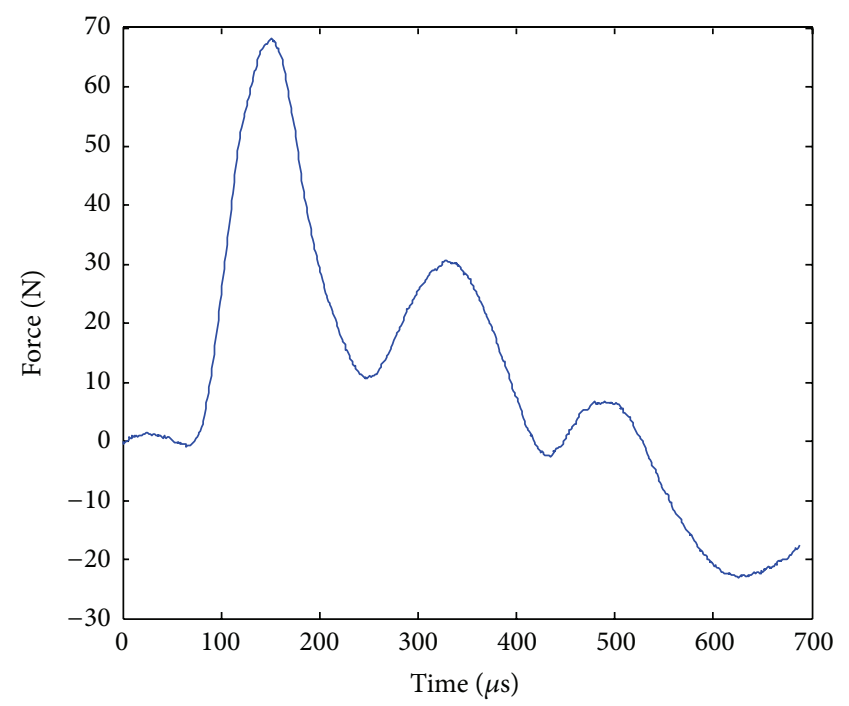

Figure 14: Typical fracture force for A1200 foam subjected to dynamic three-point bending test.

is performed. Furthermore, the fracture properties and the effect of density and strain rate on the fracture behavior are investigated.

(i) For specimens with edge cracks and circular holes, the net-section strength showed a minimal variation compared with the virgin specimen, the net-section strength is slightly higher in the case of a specimen with a center hole and slightly lower in the case of a specimen with an edge crack compared with the specimen without defect.

(ii) The fracture toughness increases as the density increases.

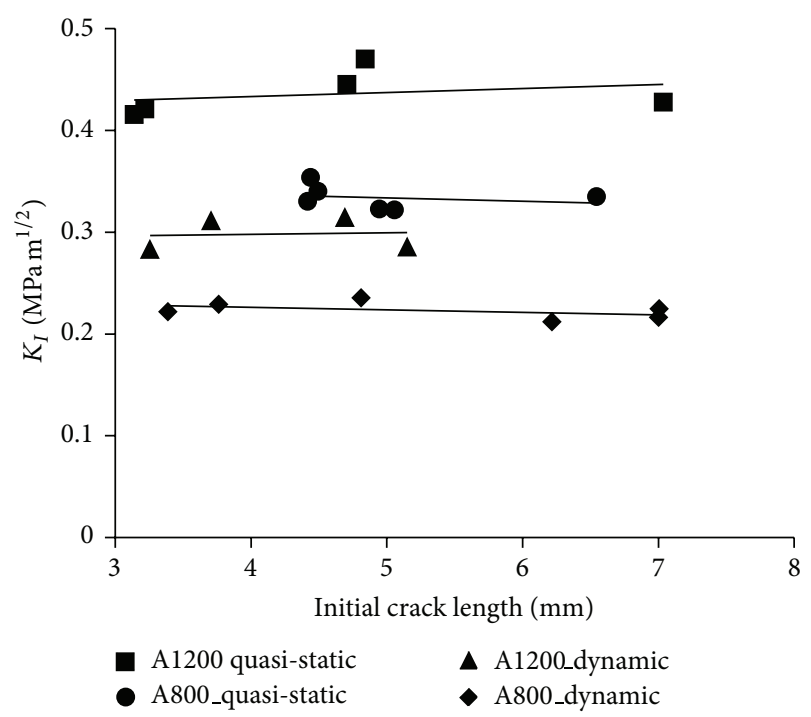

FIGURE 15: Quasi-static and dynamic fracture intensity factor as a function of initial crack length for A800 and A1200 foam (sample type 2).

(iii) The dynamic fracture toughness is lower than the quasi-static fracture toughness, which could be due to the fact that the PVC foam becomes brittle during dynamic loading.

\section{Acknowledgments}

Professor G. Ravichandran (Caltech) is gratefully acknowledged for the valuable discussions and his useful suggestions. The author thanks Gurit SP Technology for providing the material. The financial support from the University of South Carolina grant start-up fund is greatly acknowledged.

\section{References}

[1] M. E. Kabir, M. C. Saha, and S. Jeelani, "Tensile and fracture behavior of polymer foams," Materials Science and Engineering A, vol. 429, no. 1-2, pp. 225-235, 2006.

[2] E. Wang, N. Gardner, and A. Shukla, "The blast resistance of sandwich composites with stepwise graded cores," International Journal of Solids and Structures, vol. 46, no. 18-19, pp. 3492-3502, 2009.

[3] L. J. Gibson and M. F. Ashby, Cellular Solids: Structure and Properties, Cambridge University, Oxford, UK, 2nd edition, 1997.

[4] A. McIntyre and G. E. Anderton, "Fracture properties of a rigid polyurethane foam over a range of densities," Polymer, vol. 20, no. 2, pp. 247-253, 1979.

[5] C. W. Fowlkes, "Fracture toughness tests of a rigid polyurethane foam," International Journal of Fracture, vol. 10, no. 1, pp. 99-108, 1974.

[6] M. E. Kabir, M. C. Saha, and S. Jeelani, "Tensile and fracture behavior of polymer foams," Materials Science and Engineering A, vol. 429, no. 1-2, pp. 225-235, 2006.

[7] M. A. El-Hadek and H. V. Tippur, "Dynamic fracture behavior of syntactic epoxy foams: optical measurements using coherent 
gradient sensing," Optics and Lasers in Engineering, vol. 40, no. 4, pp. 353-369, 2003.

[8] F. W. Noble and J. Lilley, "Fatigue crack growth in polyurethane foam," Journal of Materials Science, vol. 16, no. 7, pp. 1801-1808, 1981.

[9] T. Cotgreave and J. B. Shortall, "The fracture toughness of reinforced polyurethane foam," Journal of Materials Science, vol. 13, no. 4, pp. 722-730, 1978.

[10] T. C. Cotgreave and J. B. Shortall, "The mechanism of reinforcement of polyurethane foam by high-modulus chopped fibres," Journal of Materials Science, vol. 12, no. 4, pp. 708-717, 1977.

[11] H. Fusheng and Z. Zhengang, "The mechanical behavior of foamed aluminum," Journal of Materials Science, vol. 34, no. 2, pp. 291-299, 1999.

[12] E. Amsterdam, P. R. Onck, and J. T. M. de Hosson, "Fracture and microstructure of open cell aluminum foam," Journal of Materials Science, vol. 40, no. 22, pp. 5813-5819, 2005.

[13] B. John, C. P. R. Nair, K. A. Devi, and K. N. Ninan, "Effect of low-density filler on mechanical properties of syntactic foams of cyanate ester," Journal of Materials Science, vol. 42, no. 14, pp. 5398-5405, 2007.

[14] E. Woldesenbet, N. Gupta, and A. Jadhav, "Effects of density and strain rate on properties of syntactic foams," Journal of Materials Science, vol. 40, no. 15, pp. 4009-4017, 2005.

[15] L. Liu and F. H. Samuel, "Effect of inclusions on the tensile properties of $\mathrm{Al}-7 \% \mathrm{Si}-0.35 \% \mathrm{Mg}$ (A356.2) aluminium casting alloy," Journal of Materials Science, vol. 33, no. 9, pp. 2269-2281, 1998.

[16] L. Marsavina, E. Linul, T. Voiconi, and T. Sadowski, "A comparison between dynamic and static fracture toughness of polyurethane foams," Polymer Testing, vol. 32, pp. 673-680, 2013.

[17] V. Rizov, "Characterization of low-velocity impact fracture behavior of rigid foam by single edge notched bend specimens," Solids and Structures, vol. 2, no. 1, pp. 1-8, 2013.

[18] E. W. Andrews and L. J. Gibson, "The influence of cracks, notches and holes on the tensile strength of cellular solids," Acta Materialia, vol. 49, no. 15, pp. 2975-2979, 2001.

[19] E. W. Andrews and L. J. Gibson, "The influence of crack-like defects on the tensile strength of an open-cell aluminum foam," Scripta Materialia, vol. 44, no. 7, pp. 1005-1010, 2001.

[20] A. Paul, T. Seshacharyulu, and U. Ramamurty, "Tensile strength of a closed-cell Al foam in the presence of notches and holes," Scripta Materialia, vol. 40, no. 7, pp. 809-814, 1999.

[21] N. A. Fleck, O. B. Olurin, C. Chen, and M. F. Ashby, "The effect of hole size upon the strength of metallic and polymeric foams," Journal of the Mechanics and Physics of Solids, vol. 49, no. 9, pp. 2015-2030, 2001.

[22] K. R. Mangipudi and P. R. Onck, "Notch sensitivity of ductile metallic foams: a computational study," Acta Materialia, vol. 59, no. 19, pp. 7356-7367, 2011.

[23] J. W. Dally and R. J. Sanford, "Strain-gage methods for measuring the opening-mode stress-intensity factor, KI," Experimental Mechanics, vol. 27, no. 4, pp. 381-388, 1987.

[24] J. R. Berger and J. W. Dally, "An overdeterministic approach for measuring KI using strain gages," Experimental Mechanics, vol. 28, no. 2, pp. 142-145, 1988.

[25] T. L. Anderson, Fracture Mechanics: Fundamentals and Applications, Taylor and Francis Group, 3rd edition, 2005.

[26] L. Rubio, J. Fernández-Sáez, and C. Navarro, "Determination of dynamic fracture-initiation toughness using three-point bending tests in a modified hopkinson pressure bar," Experimental Mechanics, vol. 43, no. 4, pp. 379-386, 2003.
[27] A. Kidane and A. Shukla, "Quasi-static and dynamic fracture initiation toughness of $\mathrm{Ti} / \mathrm{TiB}$ layered functionally graded material under thermo-mechanical loading," Engineering Fracture Mechanics, vol. 77, no. 3, pp. 479-491, 2010.

[28] L. Wang, K. Labibes, Z. Azari, and G. Pluvinage, "Generalization of split Hopkinson bar technique to use viscoelastic bars," International Journal of Impact Engineering, vol. 15, no. 5, pp. 669-686, 1994.

[29] H. Zhao, G. Gary, and J. R. Klepaczko, "On the use of a viscoelastic split Hopkinson pressure bar," International Journal of Impact Engineering, vol. 19, no. 4, pp. 319-330, 1997.

[30] A. Sharma and A. Shukla, "Mechanical characterization of soft materials using high speed photography and split hopkinson pressure bar technique," Journal of Materials Science, vol. 37, no. 5, pp. 1005-1017, 2002.

[31] D. T. Casem, W. Fourney, and P. Chang, "Wave separation in viscoelastic pressure bars using single-point measurements of strain and velocity," Polymer Testing, vol. 22, no. 2, pp. 155-164, 2003. 

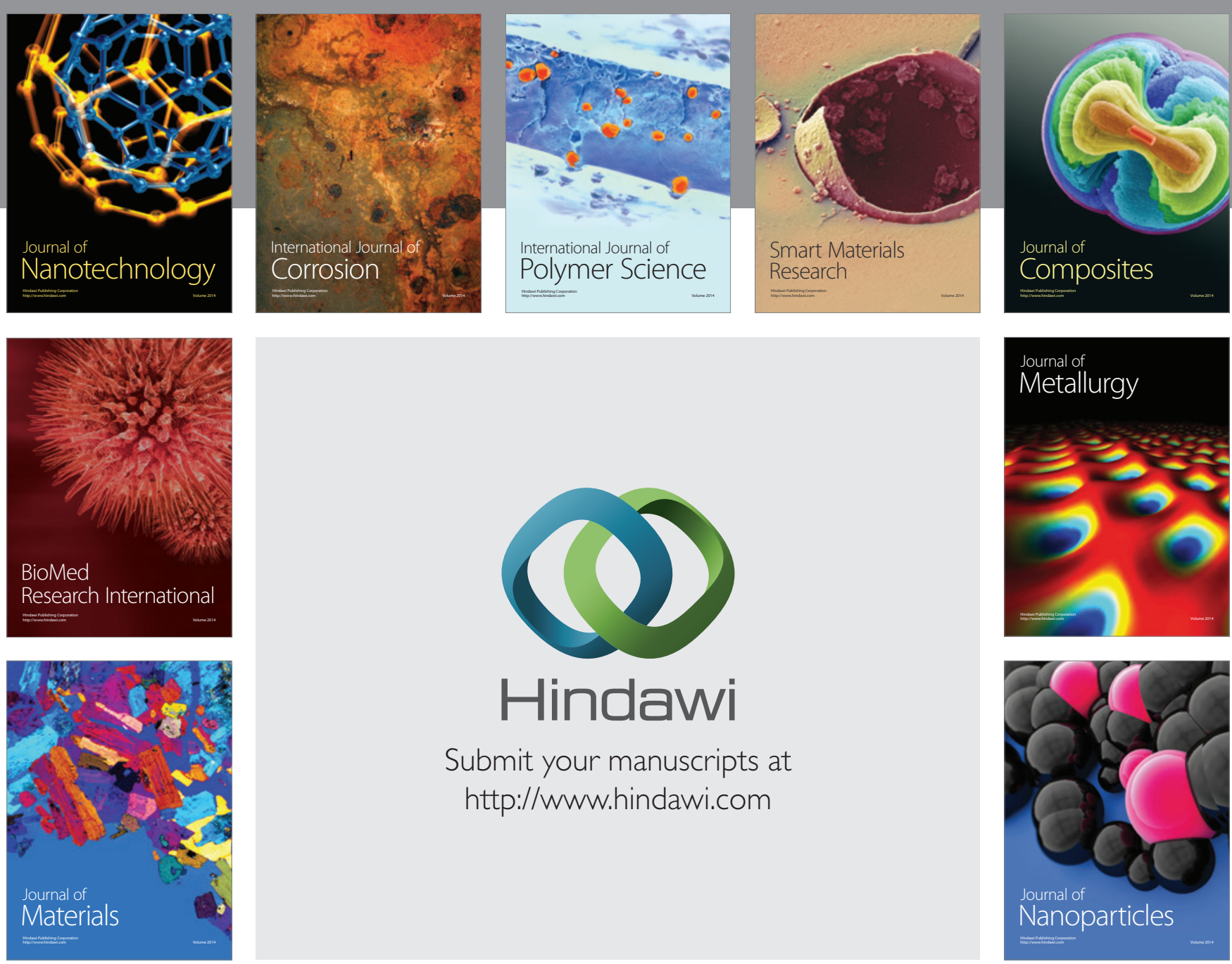

Submit your manuscripts at http://www.hindawi.com
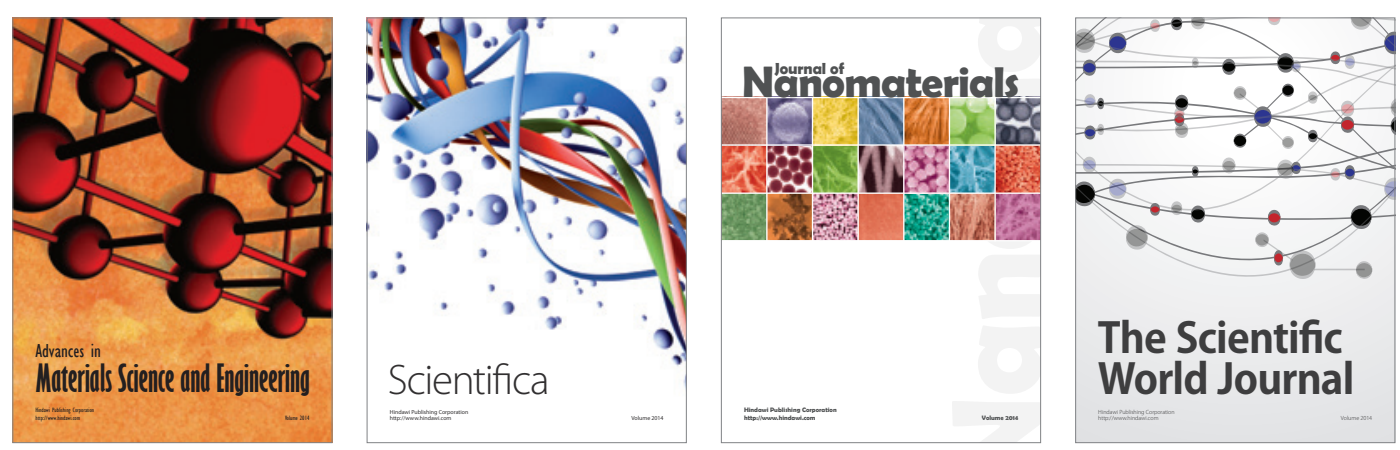

\section{The Scientific World Journal}
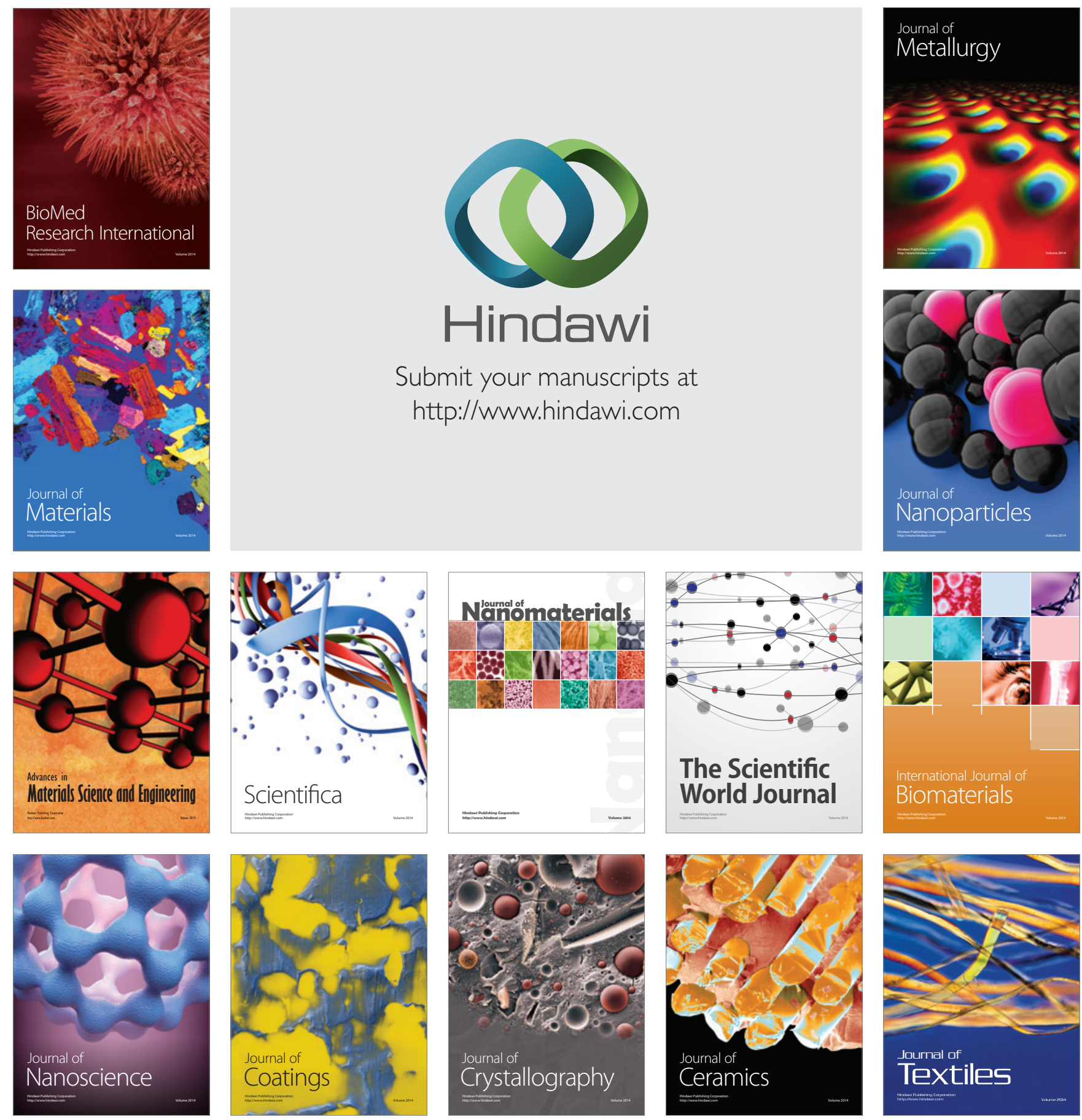